\title{
Monitoreo del manglar de Gandoca, Costa Rica (sitio CARICOMP)
}

\author{
Ana C. Fonseca E., Jorge Cortés \& Priscilla Zamora ${ }^{\dagger}$ \\ Centro de Investigación en Ciencias del Mar y Limnología (CIMAR), Ciudad de la Investigación, Universidad de \\ Costa Rica (UCR) 2060 San José, Costa Rica; afonseca@cariari.ucr.ac.cr
}

Recibido 03-XI-2005. Corregido 18-VII-2006. Aceptado 08-XII-2006.

\begin{abstract}
Monitoring of the mangrove forest at Gandoca, Costa Rica (CARICOMP site). The mangrove forest at Gandoca, Refugio Nacional de Vida Silvestre Gandoca-Manzanillo, Caribbean coast of Costa Rica, has been monitored since 1999, following the CARICOMP protocol. The dominant species was the red mangrove, Rhizophora mangle. The peak of productivity and flowering was in July. The mangrove productivity decline from 2001 to 2004 while the temperature rised. Biomass $\left(14 \mathrm{~kg} / \mathrm{m}^{2}\right)$ and density $\left(9\right.$ trees $\left./ 10 \mathrm{~m}^{2}\right)$ in Gandoca were relatively low compared to other CARICOMP sites, while productivity in July in Costa Rica ( $4 \mathrm{~g} / \mathrm{m}^{2} /$ day) was intermediate, similar to most CARICOMP sites. Rev. Biol. Trop. 55 (1): 23-31. Epub 2007 March. 31 .
\end{abstract}

Key words: mangrove, Rhizophora mangle, monitoring, CARICOMP, Gandoca, Caribbean, Costa Rica.

Los bosques de manglar están dentro de los ecosistemas costeros tropicales más productivos del mundo (Lacerda et al. 2001). En Costa Rica la cobertura de manglar está estimada en 39000 ha, el 99\% se encuentra en la costa Pacífica y el resto en la costa Caribe (Pizarro y Angulo 1993). En éste último, su distribución es incierta. En la literatura existen reportadas asociaciones de manglar en Cahuita (Malavassi et al. 1986) y Moín (Pool et al. 1977) mientras que el manglar de Gandoca es el único reconocido como bosque de manglar (Coll et al. 2001). A pesar de ello, en los mapas de humedales se destaca solo el manglar de Moín (Bravo y Ocampo 1993) o sólo el manglar de la Laguna de Gandoca y otros pequeños parches de manglar ubicados a lo largo de la costa (Córdoba Muñoz et al. 1998). En todo caso, la información existente es limitada y se desconoce su estado actual.

Aparentemente, el manglar de Gandoca es el manglar más grande, más desarrollado y en mejor estado del Caribe. Este además ha sido el más estudiado de la zona (Cortés 1991,
Coll et al. 2001, 2004), está protegido por el Refugio de Vida Silvestre Gandoca-Manzanillo (REGAMA) y ha sido designado de especial importancia internacional (RAMSAR Report for Gandoca-Manzanillo 1995).

Los manglares de la Laguna de Gandoca, en el Caribe, y el de Corcovado en el Pacífico sur de Costa Rica, son los únicos dos manglares donde se encuentra preservada la continuidad entre manglar y bosque tropical lluvioso (Jiménez y Soto 1985, Cortés 1991). La laguna de Gandoca es producto de una depresión topográfica en la interfase tierra-mar creada por interacciones fluviales y de marea (Cortés 1998). Los niveles de contaminación en la laguna son muy bajos (Coll et al. 2004). Esta laguna es un hábitat clave para varias especies de agua dulce como cangrejos, algas, esponjas y ascídeas, y es uno de los sitios principales de crianza del sábalo Megalops atlanticus (Chacón 1993) y el manatí Trichechus manatus (TNC 1998) en América Central. Además tiene el único banco natural de la ostra de mangle Crassostrea rhizophorae en el Caribe de Costa 
Rica (Cabrera et al. 1983). No hay arrecifes de coral, ni pastos marinos cercanos a este manglar. Las playas tienen oleaje intenso y son sitios importantes de anidamiento para tortugas marinas (Chacón-Chaverri 1999).

El área del manglar de Gandoca se triplicó de 4.1 ha en 1976 a 12.5 ha en 2000. Las posibles causas incluyen la sedimentación y el terremoto de 1991, que al parecer hundió el área de la laguna e hizo que el nivel del mar aumentara (Denyer 1998, Coll et al. 2001). Sin embargo, en el resto del Caribe americano la tasa anual de pérdida del manglar es del 1\% (Ellison y Farnsworth 1996). Los impactos humanos en estos humedales, especialmente conversión del manglar para maricultura, agricultura, urbanización y deforestación, son su principal amenaza (Valiela et al. 2001). Con la inquietud de evaluar el estado de los manglares del Caribe de Costa Rica se estableció una estación de monitoreo en Gandoca siguiendo el protocolo del Programa Regional "Productividad Marino Costera del Caribe (CARICOMP)". El objetivo de este manuscrito es presentar los resultados del monitoreo de biomasa, productividad, estructura forestal y condición de plántulas del mangle rojo Rhizophora mangle, especie dominante en Gandoca.

\section{MATERIALES Y MÉTODOS}

\section{Descripción del sitio}

El REGAMA está localizado en el extremo sureste de la vertiente Caribe de Costa Rica. Está rodeado por el río Sixaola en la frontera con Panamá hacia el este, una fila montañosa de la cordillera de Talamanca hacia el sur y está bañado en su lado norte por el mar Caribe. Este refugio fue creado en 1985 y tiene un territorio de 5013 ha terrestres y 4436 ha marinas, $80 \%$ es privado y $20 \%$ es propiedad del gobierno.

El clima consiste de dos estaciones lluviosas, una de noviembre a marzo y otra de junio a agosto. La tasa de precipitación anual en esta sección sur del Caribe es menor $(2500 \mathrm{~mm})$ que en la sección norte $(5000 \mathrm{~mm})$. El micro-clima dentro del Refugio se caracteriza por lluvias nocturnas y amplios períodos de sol durante el día (Herrera 1984). Las mareas son mixtas, principalmente diurnas, con un ámbito entre 30 y $50 \mathrm{~cm}$. Las corrientes fluyen de noroeste a sureste, con pequeños remolinos en dirección opuesta (Cortés 1994).

El manglar está localizado en la sección sureste del refugio, alrededor de la laguna de Gandoca. El área de la laguna y la vegetación circundante es de 266 ha, y sólo el manglar tiene un área de 12.5 ha. La especie dominante es el mangle rojo Rhizophora mangle, con Avicennia germinans (mangle negro), Laguncularia racemosa (mangle blanco) y Conocarpus erecta (botoncillo) también presentes. Tierra adentro los manglares gradualmente dan paso al bosque tropical lluvioso (Coll et al. 2001). El uso de la tierra en áreas adyacentes incluye plantaciones de banano y silvicultura. En la costa, playas de arena se alternan con acantilados rocosos formados por arrecifes de coral fósiles y rocas sedimentarias ("beach rocks") (Cortés \& Jiménez 2003).

El intercambio entre la laguna y el océano depende de la precipitación y la escorrentía. Es mayor la salida de agua dulce que la entrada de agua salada. La salida de la laguna varía de tamaño, algunas veces se encuentra cerrada y otras veces es de varios metros de ancho, fenómeno que afecta el nivel del agua en el suelo del manglar a lo largo del año.

\section{Métodos}

Con el objetivo de comparar los resultados de monitoreo con los de otros sitios en el Caribe, se siguió la metodología de CARICOMP (Wiebe et al. 1997, CARICOMP: http://isis.uwimona.edu.jm/centres/cms/caricomp/carinew.htm).

El sitio de manglar específico para el monitoreo estaba localizado en un área accesible, cerca del borde entre el manglar y el cuerpo de agua de la laguna, a $500 \mathrm{~m}$ de la boca de laguna Gandoca (9॰35'23” N; 82³5'54'W, Fig. 1). En 1999, se establecieron tres parcelas de 10 x $10 \mathrm{~m}$, para evaluar biomasa, productividad, 


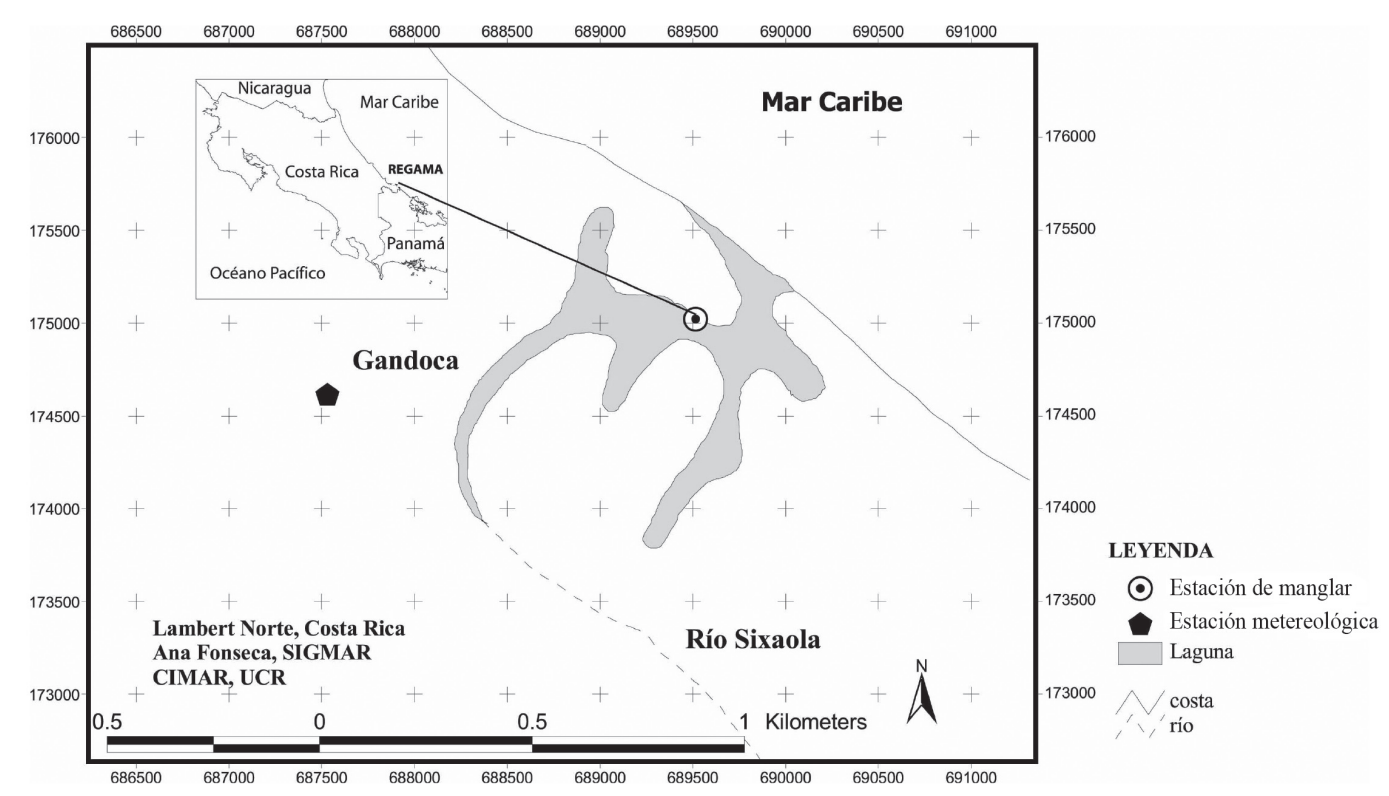

Fig. 1. Mapa de localización del sitio CARICOMP, Gandoca.

estructura forestal y estado de las plántulas. Se tenía un sensor de registro continuo de temperatura sub-acuático instalado entre las raíces del manglar, a una profundidad de $0.1 \mathrm{~m}$. La estación meteorológica para medir temperatura atmosférica y precipitación se encuentra en la casa más cercana a la entrada principal de la laguna $\left(9^{\circ} 35^{\prime} 11.2^{\prime \prime} \mathrm{N} ; 82^{\circ} 36^{\prime} 04.1\right.$ ' W), propiedad de Aquiles Rodríguez, un miembro activo de la comunidad de Gandoca y voluntario de CARICOMP. Desafortunadamente, las parcelas se perdieron durante las inundaciones de enero de 2005, causadas por una fuerte tormenta, por lo que nuevas parcelas tendrán que ser instaladas en el futuro para poder continuar con el monitoreo, lo cual implicará un cambio de sitio.

Durante el primer año de monitoreo se tomaron muestras de productividad una vez al mes de junio de 1999 a marzo de 2000, excepto en abril y mayo de 2000, para determinar el pico más alto. Julio mostró la productividad más alta, por lo que ésta fue evaluada de nuevo en julio de 2000, 2001 y 2004. Al mismo tiempo, la comunidad local fue educada sobre la importancia de los manglares y los voluntarios fueron entrenados para vigilar los experimentos y asistir en el proceso de muestreo, especialmente la recolección de las hojas para medir productividad y las medidas de temperatura y precipitación.

Las variables para determinar la estructura forestal y la productividad fueron transformadas a una distribución normal con el logaritmo en base 10. Luego de aplicar Análisis Múltiple de Varianzas Factorial (MANOVA), no se encontró interacción entre las variables independientes "parcela" y "fecha" por lo que el efecto principal de "fecha" fue analizado con Análisis Univariado (ANOVA). Los datos de productividad estacional no se pudieron normalizar por lo que se analizaron con la prueba no parámetrica de Kruskall Wallis (H) (Underwood, 1997).

\section{RESULTADOS}

\section{Estructura forestal}

Las variables medidas para determinar la estructura forestal no mostraron diferencias significativas entre los años evaluados $(\mathrm{F}=0.32$; $\mathrm{gl}=4,160 ; \mathrm{p}>0.05$, Cuadros 1 y 2 ). 
CUADRO 1

Dimensiones de los árboles del mangle rojo Rhizophora mangle en Gandoca, de 1999 a 2001 (n=28)

$\begin{array}{lccccc} & \begin{array}{c}\text { Circumferencia } \\ \text { a la altura del } \\ \text { pecho }(\mathrm{cm})\end{array} & \begin{array}{c}\text { Raíces aéreas } \\ (\mathrm{m})\end{array} & \begin{array}{c}\text { Largo del tronco } \\ (\mathrm{m})\end{array} & \begin{array}{c}\text { Altura del árbol } \\ (\mathrm{m})\end{array} & \text { Diámetro }(\mathrm{m}) \\ 1999 & 44.44 \pm 31.62 & 2.29 \pm 1.17 & 3.23 \pm 2.23 & 12.33 \pm 3.01 & 14.14 \pm 10.07 \\ 2000 & 47.61 \pm 31.32 & 2.13 \pm 1.19 & - & 11.50 \pm 0.40 & 15.63 \pm 9.03 \\ 2001 & 45.99 \pm 32.03 & 2.19 \pm 1.14 & 4.07 \pm 2.90 & 11.50 \pm 3.15 & 14.64 \pm 10.20 \\ \text { Promedio total } & 46.01 \pm 31.30 & 2.20 \pm 1.16 & 2.53 \pm 2.79 & 11.78 \pm 2.23 & 14.80 \pm 9.77\end{array}$

CUADRO 2

Estructura forestal del manglar de Gandoca de 1999 a 2001

$\begin{array}{lccccc}\text { Año } & \begin{array}{c}\text { Área basal } \\ \text { promedio } \\ \left(\mathrm{m}^{2} / \mathrm{ha}\right)\end{array} & \begin{array}{c}\text { Volumen } \\ \text { promedio del } \\ \text { tronco } \\ \left(\mathrm{m}^{3}\right)\end{array} & \begin{array}{c}\text { Biomasa } \\ \text { promedio } \\ \text { Golley et al. } 1962 \\ \left(\mathrm{~kg} / \mathrm{m}^{2}\right)\end{array} & \begin{array}{c}\text { Biomasa } \\ \text { promedio } \\ \text { Cintron \& } \\ \text { Novelli } 1984 \\ \left(\mathrm{~kg} / \mathrm{m}^{2}\right)\end{array} & \begin{array}{c}\text { Densidad de } \\ \text { árboles } \\ (\# \text { de árboles/ } \\ \left.10 \mathrm{~m}^{2}\right)\end{array} \\ 1999 & 21.83 \pm 9.65 & 0.37 \pm 0.22 & 4.48 \pm 0.71 & 14.03 \pm 6.26 & 9.33 \pm 1.53 \\ 2000 & 23.86 \pm 11.41 & 0.38 \pm 0.22 & 4.80 \pm 0.85 & 14.25 \pm 6.37 & 9.33 \pm 1.53 \\ 2001 & 23.05 \pm 10.39 & 0.36 \pm 0.24 & 4.63 \pm 0.74 & 13.36 \pm 6.53 & 9.33 \pm 1.53 \\ \text { Promedio total } & 22.91 \pm 9.14 & 0.27 \pm 0.22 & 4.63 \pm 0.68 & 13.88 \pm 5.55 & 9.33 \pm 1.32\end{array}$

\section{Plántulas}

La altura y biomasa promedio de las plántulas del manglar rojo por año muestreado se presentan en el Cuadro 3. La mortalidad de plántulas de 1999 a 2000 fue de 46\%.

\section{Productividad estacional}

La productividad total $(\mathrm{H}=76.43, \mathrm{gl}=9$, $\mathrm{p}<0.05$; Fig. 2) y la producción de flores $(\mathrm{H}=128.95$, $\mathrm{gl}=9, \mathrm{p}<0.05$; Fig. 3) resultaron significativamente más altas en julio. La producción de frutos es baja y muy variable sin diferencia significativa entre los meses muestreados $(H=15.09, g l=9, p>0.05$; Fig. 4). De toda la materia orgánica que cae del dosel del manglar, $67 \%$ corresponde a hojas, $13 \%$ a flores, $7 \%$ a brácteas, $7 \%$ a frutos, $6 \%$ a ramas y $0.21 \%$ a material misceláneo. El nivel del agua en el manglar es de hasta $33 \mathrm{~cm}$ sobre el suelo y la salinidad de $7 \mathrm{ppm}$.

\section{Productividad anual}

La productividad anual fue significativamente más alta en julio de 1999 que en julio de 2001 y julio de 2004 ( $\mathrm{F}=12.34 ; \mathrm{gl}=3,96$; p $<0.05$; Cuadro 4, Apéndice 1).

\section{Temperatura del agua}

Las temperaturas del agua más altas corresponden al año 2004, y parece que están

\section{CUADRO 3}

Altura y biomasa promedio ( \pm desv. est.) de plántulas de mangle rojo en Gandoca, de 1999 a 2001

$\begin{array}{lcc} & \text { Altura }(\mathrm{cm}) & \text { Biomasa }(\mathrm{g}) \\ 1999(\mathrm{n}=85) & 45.62 \pm 17.83 & 11.14 \pm 5.86 \\ 2000(\mathrm{n}=60) & 45.22 \pm 19.83 & - \\ 2001(\mathrm{n}=80) & 44.82 \pm 19.21 & - \\ \text { Promedio total }(\mathrm{n}=225) & 45.23 \pm 18.79 & \end{array}$


CUADRO 4

Productividad anual promedio ( \pm desv. est.) del mangle rojo Rhizophora mangle en Gandoca, 1999 a 2004

\begin{tabular}{lc}
\multicolumn{1}{c}{ Año } & $\begin{array}{c}\text { Productividad } \\
\left(\mathrm{g} / \mathrm{m}^{2} / \text { día }\right)\end{array}$ \\
& $4.64 \pm 1.62$ \\
Julio $1999(\mathrm{n}=30)$ & $3.62 \pm 1.01$ \\
Julio $2000(\mathrm{n}=20)$ & $2.91 \pm 0.80$ \\
Julio $2001(\mathrm{n}=20)$ & $3.04 \pm 1.47$ \\
Julio $2004(\mathrm{n}=30)$ & $3.61 \pm 1.50$ \\
Promedio $(\mathrm{n}=100)$ & \\
& \\
aumentando, sin embargo esto podría estar rela- \\
cionado al hecho de que el sensor fue removido \\
de su posición original. Las temperaturas más \\
bajas se registraron en el 2002 (Cuadro 5).
\end{tabular}

\section{Temperatura atmosférica y precipitación}

En la atmósfera las temperaturas más altas se registraron en el 2002 y las más bajas en el
CUADRO 5

Temperatura del agua promedio ( \pm desv. est.) anual en Gandoca, de 1999 a 2004

\begin{tabular}{lccc}
\multicolumn{1}{c}{ Año } & $\begin{array}{c}\text { Temperatura } \\
\text { mínimo } \\
\text { promedio } \\
\left({ }^{\circ} \mathrm{C}\right)\end{array}$ & $\begin{array}{c}\text { Temperatura } \\
\text { máxima } \\
\text { promedio } \\
\left({ }^{\circ} \mathrm{C}\right)\end{array}$ & $\begin{array}{c}\text { Temperatura } \\
\text { promedio } \\
\left({ }^{\circ} \mathrm{C}\right)\end{array}$ \\
2000 & $22.41 \pm 0.48$ & $33.41 \pm 3.72$ & $25.83 \pm 0.94$ \\
2001 & $22.26 \pm 0.69$ & $31.64 \pm 1.46$ & $25.72 \pm 0.42$ \\
2002 & $21.99 \pm 1.07$ & $30.56 \pm 1.80$ & $25.66 \pm 0.39$ \\
2003 & $25.31 \pm 1.41$ & $34.83 \pm 1.30$ & $29.59 \pm 1.52$ \\
2004 & $24.19 \pm 1.61$ & $36.15 \pm 2.57$ & $28.17 \pm 1.48$ \\
$\begin{array}{l}\text { Promedio } \\
\text { total }\end{array}$ & $23.23 \pm 1.05$ & $33.32 \pm 2.17$ & $26.99 \pm 0.95$
\end{tabular}

temperatura del agua aparentemente aumentó, pero se necesita muestrear por un mayor período de tiempo para determinar si hay correlación entre ambos. Sin embargo, anteriormente se comprobó en un análisis integral de varios sitios CARICOMP, que la tendencia de productividad está claramente relaciona-

2001. No hay tendencias claras en los últimos 5 años. La precipitación mensual promedio fue de $268.9 \pm 175.0 \mathrm{~mm}$, es muy variable y no muestra tendencias claras. La precipitación anual promedio es de $2749.3 \mathrm{~mm}$ (Cuadro 6).

\section{DISCUSIÓN}

La especie dominante en la estación de monitoreo del manglar de Gandoca fue el mangle rojo Rhizophora mangle, al igual que el resto del área (Coll et al. 2001). El pico de productividad y producción de flores a lo largo del año ocurre alrededor de julio, dentro de la segunda estación lluviosa (Herrera 1984). La productividad del manglar disminuyó desde el 2001 y la

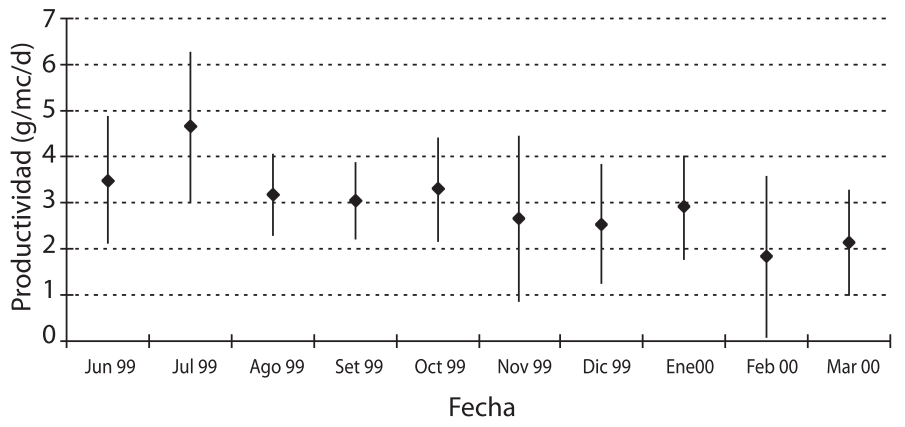

Fig. 2. Productividad total estacional de Rhizophora mangle, Gandoca, Costa Rica $(n=30)$.

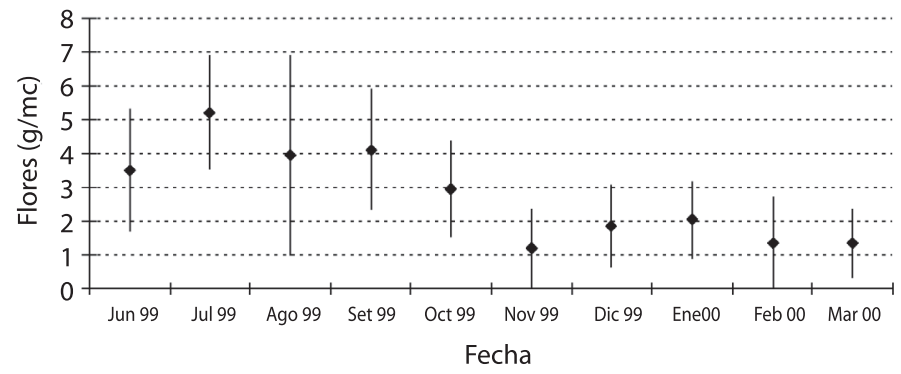

Fig. 3. Producción estacional de flores de Rhizophora mangle, Gandoca, Costa Rica $(n=30)$. 


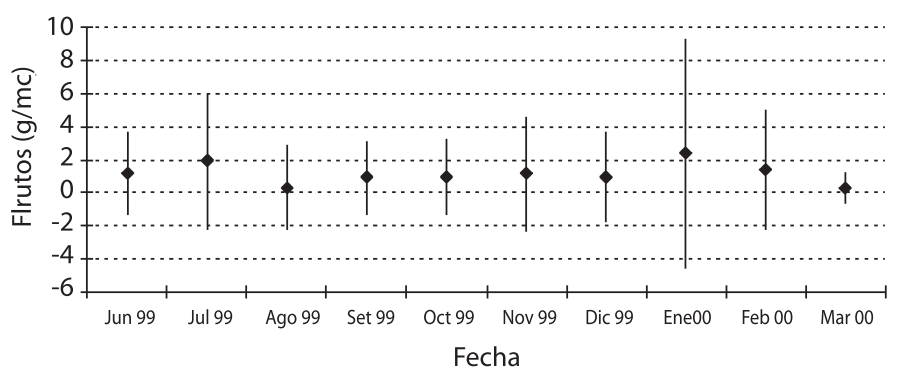

Fig. 4. Producción estacional de frutos de Rhizophora mangle, Gandoca, Costa Rica $(n=30)$. da con las temperaturas y la precipitación sin embargo se necesitan bases de datos más extensas para poder evaluar esta última relación (Wiebe et al. 1997). La precipitación anual promedio encontrada (2749.3 mm) coincide con lo reportado para la zona $(2500$ mm, Herrera 1984).

Debido a los escasos estudios realizados en el

CUADRO 6

Temperatura atmosférica y precipitación promedio ( \pm desv. est.) en Gandoca, 2000 a 2003

$\begin{array}{lccccc}\text { Año } & \begin{array}{c}\text { Temperatura } \\ \text { mínima promedio } \\ \left({ }^{\circ} \mathrm{C}\right)\end{array} & \begin{array}{c}\text { Temperatura } \\ \text { máxima promedio }\end{array} & \begin{array}{c}\text { Temperatura } \\ \text { promedio }\left({ }^{\circ} \mathrm{C}\right)\end{array} & \begin{array}{c}\text { Precipitación } \\ \text { mensual promedio } \\ (\mathrm{mm})\end{array} & \begin{array}{c}\text { Precipitación } \\ \text { anual } \\ (\mathrm{mm})\end{array} \\ 2000 & 20.54 \pm 0.91 & 33.79 \pm 0.40 & 25.80 \pm 0.29 & 213.5 \pm 167.6 & 1494.3 \\ 2001 & 20.27 \pm 1.17 & 33.16 \pm 0.56 & 25.67 \pm 0.57 & 309.7 \pm 153.8 & 2787.7 \\ 2002 & 21.28 \pm 1.04 & 34.85 \pm 0.78 & - & 364.2 \pm 215.4 & 4369.8 \\ 2003 & 21.21 \pm 1.01 & 34.77 \pm 0.78 & 26.53 \pm 0.55 & 194.9 \pm 108.5 & 1948.9 \\ 2004 & 20.19 \pm 1.29 & 34.55 \pm 0.38 & 25.96 \pm 0.79 & 262.1 \pm 229.92 & 3145.6 \\ \begin{array}{l}\text { Promedio } \\ \text { total }\end{array} & 20.70 \pm 1.08 & 34.22 \pm 0.58 & 25.99 \pm 0.55 & 268.9 \pm 175.0 & 2749.3\end{array}$

Caribe costarricense es difícil comparar los valores obtenidos con otros manglares de la zona. Sin embargo, cabe mencionar que para el manglar de Moín, un manglar de tipo riverino, Pool et al. (1977) determinaron que la especie dominante era Pterocarpus officinalis. Esto difiere con lo encontrado para el manglar de Gandoca, que es lacustre y la especie dominante es el $R$. mangle. Además, para el manglar de Moín se calculó un área basal de Rhizophora de $8.0 \mathrm{~m}^{2} /$ ha, un valor considerablemente inferior al obtenido para la zona de estudio $\left(22.91 \pm 9.14 \mathrm{~m}^{2} / \mathrm{ha}\right)$.

Gandoca posee árboles de mangle con una altura promedio similar al reportado en los manglares de Tamarindo $(=10.7 \mathrm{~m})$ en el Pacífico Norte y Sierpe (10.8 m) en el Pacífico Sur de Costa Rica (Jiménez y Soto 1985). Sin embargo su área basal es menor que la reportada para estos dos manglares (Jiménez y Soto 1985), esta es más bien similar a la de los manglares de Pochote en el Pacífico Norte y Puntarenas en el Pacífico Central (Jiménez y Soto 1985) y menor que el manglar de Tivives (Jiménez 1988) ubicado en la zona seca entre el Pacífico Norte y Central. Es difícil concluir algo a este respecto, sobre todo porque en los sitios para los que existe información estos valores se calculan con base en todas las especies presentes y no solo en $R$. mangle como se hizo en el presente estudio. Siendo entonces que otras especies con mayor altura y desarrollo comunes en el Pacífico (Jiménez 1994), como lo son Rhizophora racemosa, Avicennia germinans, Avicennia bicolor y Pelliciera rhizophorae, influyen en los valores calculados para otros manglares.

Al considerar los valores de precipitación anual y de temperaturas atmosféricas (Cuadro 6), junto con valores de la estructura forestal 
se podría decir que la zona estudiada tiende a compartir valores con sitios del Pacífico costarricense que poseen índices de complejidad de Holdridge intermedios (Jiménez y Soto 1985, Jiménez 1999).

De los 11 sitios en el Caribe de monitoreo CARICOMP que publicaron datos de $R$. mangle en 1997, la biomasa en 1995, medida según la metodología de Cintrón-Molero \& Shaeffer-Novelli (1984), varió de aproximadamente $1 \mathrm{~kg} / \mathrm{m}^{2}$ en Bahamas y Carrie Bow Cay, Belice, a $19 \mathrm{~kg} / \mathrm{m}^{2}$ en Colombia y Venezuela, mientras que el número de árboles por parcela varió de 9 en Venezuela a 87 en República Dominicana (Wiebe et al. 1997). Por lo tanto, la biomasa $\left(14 \mathrm{~kg} / \mathrm{m}^{2}\right)$ y densidad (9 árboles por parcela) en Gandoca son relativamente bajas, su biomasa es similar a la encontrada en Bermuda $\left(12 \mathrm{~kg} / \mathrm{m}^{2}\right)$ y su densidad similar a la de Venezuela (9 árboles por $100 \mathrm{~m}^{2}$ ). La productividad anual varió entre aproximadamente $1 \mathrm{~g} / \mathrm{m}^{2} /$ día en Bahamas y Bermuda, a $10 \mathrm{~g} / \mathrm{m}^{2} /$ día en Venezuela. La productividad encontrada para julio en Costa Rica ( $4 \mathrm{~g} / \mathrm{m}^{2} /$ día) es similar a lo que se encontró en la mayoría de los sitios CARICOMP de dicho informe, Gran Caimán, Trinidad y Bermuda, para el mismo mes, pero menor que lo encontrado en Venezuela. En Venezuela y Gran Caimán, julio también era uno de los meses más productivos del año. Los estimados más altos de biomasa sobre el suelo de manglar dominado por Rhizophora han sido informados para Asia y el Pacífico, de hasta $70 \mathrm{~kg} / \mathrm{m}^{2}$, y una productividad promedio de $3 \mathrm{~g} / \mathrm{m}^{2} /$ día (Clough 1992). La mayoría de los datos de biomasa de CARICOMP, incluidos los de Costa Rica caen dentro de la mitad inferior del ámbito de biomasa y los de productividad se acercan al promedio global.

Los manglares actúan principalmente como exportadores de nutrientes y trampas de sedimento, no hay duda que su remoción afecta adversamente la calidad del agua (Wiebe et al. 1997). El área del manglar de Gandoca se triplicó de 1976 a 2000, posiblemente debido a procesos de sedimentación originados por la escorrentía de los ríos corriente arriba, o por el aumento en el nivel del mar producto del hundimiento de la laguna durante el terremoto de abril de 1991 (Coll et al. 2001). Aparentemente, este manglar a sufrido poco impacto por parte de la comunidad local (Coll et al. 2001, 2004), ya que el uso de la madera del manglar se ha limitado a actividades de supervivencia y las prácticas agrícolas se están reemplazando por ecoturismo en el área de la laguna. La conciencia ecológica de los habitantes de Gandoca y el estado de protección del manglar por el REGAMA podría garantizar la supervivencia, expansión y funcionalidad de este manglar, y el bienestar de la gente que depende del mismo. Se recomienda seguir actualizando la cobertura del manglar de Gandoca y buscar más profundamente las causas de su beneficiosa expansión.

\section{AGRADECIMIENTOS}

Este proyecto fue apoyado por CARICOMP, CONICIT, UNEP, US-ISRAEL Cooperative Development Research Program y la Vicerrectoría de Investigación de la Universidad de Costa Rica. Gracias infinitas a Eva Salas, Marta Coll, Pablo Sánchez, Paula González, José Luis Velázquez, Aquiles Rodríguez y su familia, la Asociación de Desarrollo y el Comité Zonal de Gandoca, y toda la gente del REGAMA por su participación.

\section{RESUMEN}

El manglar de Gandoca, Refugio Nacional de Vida Silvestre Gandoca-Manzanillo, Caribe de Costa Rica, se ha monitoreado desde 1999. La especie dominante es el mangle rojo Rhizophora mangle. El pico de productividad y producción de flores a lo largo de los años se dio en julio. La productividad del manglar disminuyó desde el 2001 y la temperatura del agua aparentemente aumentó. La biomasa 
$\left(14 \mathrm{~kg} / \mathrm{m}^{2}\right)$ y densidad (9 árboles por $100 \mathrm{~m}^{2}$ ) en Gandoca son relativamente bajas comparados con otras manglares dentro del Programa CARICOMP, mientras que la productividad encontrada para julio en Costa Rica ( $4 \mathrm{~g} / \mathrm{m}^{2} /$ día $)$ es intermedia, similar a lo que se encontró en la mayoría de los sitios CARICOMP.

Palabras clave: manglar, Rhizophora mangle, monitoreo, CARICOMP, Gandoca, Caribe, Costa Rica.

\section{REFERENCIAS}

Cabrera, P.J., E. Zamora M. \& O. Pacheco. 1983. Crecimiento y madurez sexual de Crassostrea rhizophorae (Guilding 1828) cultivada en sistema suspendido en Estero de Vizcaya, Limón, Costa Rica. Rev. Biol. Trop. 32: 167-169.

Chacón, D. 1993. Aspectos biométricos de una población de sábalo Megalops atlanticus (Pisces: Megalopidae). Rev. Biol. Trop. 41: 13-18.

Chacón-Chaverri，D. 1999. Anidación de la tortuga Dermochelys coriacea (Testudines: Dermochelyidae) en playa Gandoca, Costa Rica (1990 a 1997). Rev. Biol. Trop. 47: 225-236.

Cintrón-Molero, G. \& Y. Schaeffer-Novelli. 1984. Methods for studying mangrove structure. Pp. 91-113. In: S. C. Snedaker \& J. G. Snedaker (eds.). The mangrove ecosystem: Research methods. UNESCO, Bungay, Gran Bretaña, 251p

Clough, B.F. 1992. Primary productivity and growth of mangrove forests. Pp: 225-249. In A.I. Robertson \& D.M. Alongi (eds.). Tropical Mangrove Ecosystems. Am. Geophys. Union, Washington DC. Pp: 225-249.

Coll, M., A. C. Fonseca \& J. Cortés. 2001. El manglar y otras asociaciones vegetales de la laguna Gandoca, Limón, Costa Rica. Rev. Biol.. Trop. 49 (Supl. 2): 321-329.

Coll, M., J. Cortés \& D. Sauma. 2004. Características físico-químicas y determinación de plaguicidas en el agua de la laguna de Gandoca, Limón, Costa Rica. Rev. Biol.. Trop. 52 (Supl. 2): 33-42.

Córdoba Muñoz, R., J.C. Romero Araya \& N. Windevoxhel L. 1998. Inventario de humedales de Costa Rica. MINAE/SINAC-UICN/HORMA, San José, Costa Rica. 380 p.

Cortés, J. 1991. Ambientes y organismos marinos del Refugio Nacional de Vida Silvestre Gandoca Manzanillo, Limón, Costa Rica. Geoistmo 5: 62-68.
Cortés, J. 1994. A reef under siltation stress: a decade of degradation: 240-246. In: R.N. Ginsburg (compiler). Proc. Colloquium on Global Aspects of Coral Reefs: Health, Hazards and History, 1993. RSMAS, Univ. Miami, Miami, Florida.

Cortés, J. 1998. Cahuita and Laguna Gandoca, Costa Rica: 107-113. In: B. Kjerfve (ed.), CARICOMP -Caribbean Coral Reef, Seagrass and Mangrove Sites. UNESCO, Paris.

Cortés, J. \& C. E. Jiménez. 2003. Past, present and future of the coral reefs of the Caribbean coast of Costa Rica: p: 223-239. In: J. Cortés (ed.), Latin American Coral Reefs. Elsevier Science B.V., Amsterdam, Holanda.

Denyer, P. 1998. Historic-prehistoric earthquakes, seismic hazards and Tertiary and Quaternary geology of the Gandoca-Manzanillo National Wildlife Refuge, Limón, Costa Rica. Rev. Biol. Trop. 46 (Suppl. 6): 237-250.

Ellison, A.M. \& E.J. Farnsworth. 1996. Anthropogenic disturbance of Caribbean mangrove ecosystems: past impacts, present trends and future predictions. Biotropica 28: 549-565.

Golley, F., H.T. Odum, R.F. Wilson. 1962. The structure and metabolism of a Puerto Rican red mangrove forest in May. Ecology 43: 9-19.

Herrera, W. 1984. Clima de Costa Rica. Editorial Universidad Estatal a Distancia (EUNED), San José, Costa Rica, 118 p.

Jiménez, J.A. 1994. Los Manglares del Pacífico Centroamericano. EFUNA, Heredia, Costa Rica. 352 p.

Jiménez, J.A. 1988. The dynamics of Rhizophora racemosa Meyer, forests on the Pacific coast of Costa Rica. Brenesia 30: 1-12.

Jiménez, J. A. 1999. Ambiente, distribución y características estructurales en los manglares del Pacífico de Centro América: contrastes climáticos: p. 5170. In A. Yañez-Arancibia \& A.L. Lara-Domínguez (eds.) Ecosistemas de manglar en América Tropical. Instituto de Ecología, A.C. México, UICN/ORMA, Costa Rica, NOAA/NMFS Silver Spring, Maryland, EEUU. 380 p.

Jiménez, J.A. \& R. Soto. 1985. Patrones regionales en la estructura y composición florística de los manglares de la costa Pacífica de Costa Rica. Rev. Biol. Trop. 33: 25-37.

Lacerda, L.D., J.E. Conde, B. Kjerfve, R. Alvarez-León, C. Alarcón \& J. Polanía. 2001. American mangrove, pp: 
1-62. In L.D. Lacerda (ed.). Mangrove Ecosystems: Function and Management. Springer-Verlag, Berlín.

Malavassi L., R.M. Alfaro, W. Murillo \& G. Herrera. 1996. Evaluación del recurso biológico del manglar de Tivives. Fundación Parques Nacionales, Programa de Patrimonio Natural de Costa Rica, San José, Costa Rica. 48 p.

Pizarro, F. \& H. Angulo. 1993 Diagnóstico de los manglares de la costa Pacífica de Costa Rica: Informe para la Comisión Nacional de Manglares. UICN, San José, Costa Rica. 82 p

Pool, D.J., S.C. Snedaker \& A.E. Lugo. 1977. Structure of mangrove forests in Florida, Puerto Rico, Mexico, and Costa Rica. Biotropica 9:195-212.

TNC. 1998. Evaluación ecológica rápida del Corredor Biológico Talamanca-Caribe. ProAmbiente/The Nature Conservancy, Washington, D.C. 226 p.

Underwood, A. 1997. Experiments in ecology: their logical design and interpretation using analysis of variance. Univ. Cambridge, Cambridge, Inglaterra. 504 p.

Valiela, I. J.L. Bowen \& J.K. York. 2001. Mangrove forests: one of the world's threatened major tropical environments. BioScience 51: 807-815.

Wiebe, W.J., D.T. Gerace, L. Flowers, L. Johnson, C. Ward, H. Oxenford, C. Parker, J. Tschirky, S.R. Smith, J. Ellison, D. De Meyer, P. Bush, J. Garzón-Ferreira, J.
Nivia, L.P.J.J. Por, J.A. Nagelkerken, F.X. Geraldes, J. Ramírez, J. Herrera-Silveira, R.O. Sanchez-Arguelles, J.R. Garcia, G. Alleng, K. Bonair, R. Laydoo, R. Varela, E. Klein, D. Bone, D. Perez \& D. Linton. 1997. Structure and productivity of mangrove forests in the Greater Caribbean Region. CARICOMP. Proc. of the $8^{\text {th }}$ ICRS, Panama 1: 669-672.

\section{REFERENCIAS DE INTERNET}

CARICOMP: http://isis.uwimona.edu.jm/centres/cms/caricomp/carinew.htm. Consultado 23 octubre 2006.

RAMSAR Report for Gandoca Manzanillo. 1995. Ramsar Sites Information Service. http://www.wetlands.org/ RSDB/default.htm. Consultado 23 octubre 2006.

APÉNDICE 1

Resultados de prueba Bonferroni para deteminar diferencias de productividad significativas entre los años muestreados

$\begin{array}{lcccc} & \text { Jul-00 } & \text { Jul-01 } & \text { Jul-04 } & \text { Jul-99 } \\ \text { Jul-00 } & 1.00 & & & \\ \text { Jul-01 } & 0.29 & 1.00 & & \\ \text { Jul-04 } & 0.16 & 1.00 & 1.00 & \\ \text { Jul-99 } & 0.06 & 0.00 & 0.00 & 1.00\end{array}$


